\title{
Europas Energiesicherheit: zwischen Binnenmarkt, Interdependenz und strategischen Herausforderungen
}

\author{
Johannes Pollak*
}

The attempts of the European Union (EU) to create energy security have always oscillated between a strong liberal market approach and a more security oriented one. The former builds on the principles of a single market for energy products to the advantage of the consumer while the latter treats energy as a commodity best understood as a strategic good, i.e. a national commodity. Recently, this dichotomy has been joined by concerns over the devastating effects of $\mathrm{CO}_{2}$ emissions and the resulting global warming. The challenges the EU is facing can hardly be underestimated: decreasing its dependence on Russian imports without destabilizing its Eastern neighbour, convincing the member states of the advantages of a stronger cooperation in the energy field, creating a joint energy vision, establishing a resilient infrastructure for energy supply within and beyond the EU's borders, and fulfilling the objectives of the Paris Agreement, to name but a few. This contribution sheds light on the concept of energy security and discusses the current state of Europe's energy security.

Langjährigen Beobachtern der europäischen Energiepolitik fällt es nicht schwer, drei Konstanten einer mühsamen Entwicklung auszumachen: erstens die Europäische Kommission - bzw. in den Anfangsjahren der europäischen Integration die Hohe Behörde der Europäischen Gemeinschaft für Kohle und Stahl - als treibende Kraft, die entgegen allen Widerständen der Mitgliedstaaten seit den Gründungsjahren versucht, einen Binnenmarkt für Kohle, Gas und Erdöl zu etablieren. In den 1950er und 1960er Jahren war die gesamte Aufmerksamkeit auf den Wiederaufbau der durch den Zweiten Weltkrieg zerstörten Infrastruktur gerichtet. Die Ausbildung einer grenzüberschreitenden Infrastruktur und des grenzüberschreitenden Handels war (und ist) ein geeignetes Mittel, nationale Monopole $\mathrm{zu}$ brechen und Versorgungssicherheit herzustellen. Mit dem Vertrag von Lissabon und seinem Energiekapitel, welches die Ziele der Energiepolitik „im Geiste der Solidarität zwischen den Mitgliedstaaten "1 definiert, wurde die Primärrechtsentwicklung vorangetrieben. Unermüdlich versucht die Kommission, mit Grün- und Weißbüchern die Energiepolitik weiterzuentwickeln.

Zweitens die Bedeutung externer Schocks für die integrative Weiterentwicklung eines Politikfeldes, das traditionell aus sicherheitspolitischen nationalen Interessen mehr der Abschottung als der Öffnung verpflichtet war: Der erste Ölpreisschock 1973 sowie der russisch-ukrainische Gastransitstreit seit dem Jahr 2005 verdeutlichen dies besonders. Ein weiteres Beispiel für die Bedeutung externer Faktoren ist der Preis des jeweiligen Energieträgers. US-amerikanisches Fracking-Gas beeinflusst die Verfügbarkeit kostengünstiger Energie ebenso wie die australischen Pläne zum Export von Flüssiggas oder russische Ankündigungen einer Energiekooperation mit der Volksrepublik China. Der europäische de-regulatorische Wille scheint proportional zur Höhe des Öl- und Gaspreises: Je geringer

\footnotetext{
* Prof. Dr. Johannes Pollak, Rektor der Webster Vienna Private University, Wien.

1 Konsolidierte Fassung des Vertrags über die Arbeitsweise der Europäischen Union (AEUV) in der Fassung des Vertrags von Lissabon, Art. 122, in: Amtsblatt der EU, Nr. C 83 vom 30. März 2010, S. 47-388, hier S. 98.
} 
die Energiekosten, desto leiser wurden die Stimmen in den Mitgliedstaaten der Europäischen Union (EU), die mehr Wettbewerb im Energiesektor oder die Reduktion fossiler Energieträger zur Stromgewinnung forderten.

Die dritte Konstante ist die immer größere Bedeutung des Klima- und Umweltschutzes. ${ }^{2}$ Bereits die Einheitliche Europäische Akte nahm den Umweltschutz in das Primärrecht der Gemeinschaft auf. In der Folge konnten energiepolitisch relevante Rechtsakte über den Umweg des Umweltschutzes erlassen werden. Zwischenzeitlich haben sich sowohl die primär- als auch sekundärrechtliche Basis verändert und der Klima- und Umweltschutz wurde als eindeutige Priorität anerkannt. Insbesondere die Richtlinie 2003/87/EG vom 13. Oktober 2003 über ein System für den Handel mit Treibhausgasemissionszertifikaten war ein wichtiger Schritt, um die $\mathrm{CO}_{2}$-Emissionen als gravierendes Problem im öffentlichen Diskurs zu verankern. Das Pariser Übereinkommen der Klimarahmenkonvention der Vereinten Nationen (United Nations Framework Convention on Climate Change, UNFCCC) von 2015 dient der Europäischen Kommission als wichtiger Referenzpunkt für die Erreichung klimapolitischer Ziele. Es bietet zudem die Möglichkeit, die EU als Vorreiter in Sachen Klimapolitik zu etablieren. Inwieweit dies bisher eher auf der symbolischen denn auf der faktischen Ebene passiert ist, ist Gegenstand vieler Diskussionen.

Bevor im Folgenden auf die Frage der Energiesicherheit Europas und auf die (mangelnde) strategische EU-Energiepolitik eingegangen wird, wird in einem ersten Teil die Energieunion als politischer Hintergrund eingeführt und danach das Konzept der Energiesicherheit diskutiert. Der Beitrag wirft ein Schlaglicht auf folgende Aspekte: die Komplexität einer Energiesicherheitspolitik, die Interdependenz von Energiebeziehungen und die strategische Planung von Energiesicherheit. Klar ist, dass eine Energiesicherheitspolitik im 21. Jahrhundert ohne den Klima- und Umweltschutz als zentrales Thema nicht nur anachronistisch, sondern weitestgehend sinnlos ist.

\section{Die Energieunion - mehr als eine neue Verpackung?}

Die Europäische Kommission übernimmt weiterhin die Rolle des Initiators für die weitere Integration der Energiepolitik. Symbolisch bedeutend war die von Jean-Claude Juncker ausgerufene Energieunion als eine von zehn Prioritäten der Europäischen Kommission 2014-2019.3 Anstelle verschiedener Liberalisierungspakete und ihrer schleppenden Implementierung, der mangelnden strategischen Ausrichtung und der schleichenden Renationalisierung sollte die Energieunion nicht nur einen neuen Aufbruch signalisieren - im Übrigen an die Zeit der Kohle- und Stahlunion erinnernd -, sondern vor allem alle Bereiche der bisherigen europäischen Klima- und Energiepolitik in einem kohärenten Rahmen umfassen. Aus der lange diskutierten Energiegemeinschaft wurde die Energieunion. Die Rede der neuen Kommissionspräsidentin Ursula von der Leyen vor dem Europäischen Parlament am 16. Juli 2019 - zu dem Zeitpunkt noch als Kandidatin für das Amt - lässt auch an der zukünftigen Bedeutung der Klima- und Umweltschutzpolitik der Europäischen Kommission keinen Zweifel. Es ist nicht lediglich eine Fortführung der bisherigen Politik zu erwarten. Vielmehr wird die Intensivierung der Anstrengungen, die Mitgliedstaaten auf

2 Siehe Johannes Pollak/Samuel R. Schubert/Peter Slominski: Die Energiepolitik der EU, Wien 2010.

3 Europäische Kommission: Zehn Prioritäten für Europa. Ein neuer Start für Europa: eine EU-Agenda für Jobs, Wachstum, Fairness und demokratischen Wandel, Brüssel 2015, S. 6. 
einen gemeinsamen Kurs zu verpflichten, als eine Hauptaufgabe für die nächsten Jahre definiert. ${ }^{4}$

Ein Vorteil des griffigen Schlagworts der Energieunion war und ist, dass sich der Inhalt fast beliebig interpretieren lässt. In den vergangenen Jahren prägten die Ukrainekrise und die damit verbundenen realen oder auch fiktiv überhöhten Lieferengpässe von Erdgas die öffentliche Wahrnehmung der Energiesicherheit Europas. War für die einen die Energieunion im Wesentlichen ein sicherheitspolitisch motiviertes Unternehmen, sahen andere darin eine Chance auf Vollendung des europäischen, liberalisierten Energiebinnenmarktes. Diese Dichotomie wurde in jüngster Zeit durch die neue Aufmerksamkeit für den Klimaund Umweltschutz ergänzt. Lässt das breite Konzept die östlichen Mitgliedstaaten vor allem in Kategorien der Versorgungssicherheit und Abhängigkeit von Russland denken, erlaubt es den westlichen und nördlichen Mitgliedstaaten, den Fokus auf den Klimaschutz zu legen. Erstaunlich ist allerdings, dass die Energieunion kaum mehr als alter Wein in neuen Schläuchen war. Die Kommission unter Präsident Juncker, die mit der Ernennung eines Vizepräsidenten für die Energieunion zwar ein Signal für die politische Bedeutung des Themas setzte, verließ sich in der Ausgestaltung konkreter Schritte auf Althergebrachtes. Damit wollte man ein Ausbrechen alter Konflikte zwischen den Mitgliedstaaten verhindern. Energie war und ist ein strategisches Gut und selbst nach fast 70 Jahren Integrationsbemühungen sind viele Mitgliedstaaten, vor allem in Mittel- und Osteuropa, nicht willens, mehr als einen lückenhaften Energiebinnenmarkt zu implementieren. Wo das Schlagwort „Energieunion“" also einen mutigen Aufbruch zu neuen Ufern verhieß, blieb letztendlich nüchterner Pragmatismus. Pragmatische Liberalisierungsschritte durch Harmonisierung im Binnenmarkt und die Integration der Strommärkte waren richtige und wichtige Schritte. Zu einer gesamteuropäischen strategischen Debatte kam es bisher jedoch nicht. Ein gutes Beispiel für den plakativen Charakter der Energieunion ist die Frage des gemeinsamen Gaseinkaufes der EU. Vorbild für die Idee des gemeinsamen Einkaufs war die Euratom-Versorgungsagentur, die das vorrangige Bezugsrecht für Erze, Ausgangsstoffe und besondere spaltbare Stoffe aus Staaten innerhalb oder außerhalb der EU hat. Vom Präsidenten des Europäischen Rates bereits 2014 lanciert, erschien der Vorschlag auf den ersten Blick durchaus sinnvoll, die russische Macht bei Erdgaslieferungen zu balancieren. Donald Tusk wollte den verpflichtenden gemeinsamen Gaseinkauf in mehreren Stufen verwirklicht sehen: (1) Beseitigung aller intransparenten und wettbewerbsfeindlichen Vertragsklauseln in bestehenden bilateralen Gaslieferverträgen; (2) ein einheitlicher Mustervertrag für Neuverträge; (3) eine Beteiligung der Kommission an Vertragsverhandlungen für Gaslieferungen. In der Energiewirtschaft und -ökonomie wunderte man sich dagegen über die Aushebelung fundamentaler Prinzipien des freien Handels und des Binnenmarktes. Letzterer ist nach Art. 3 Abs. 3 des Vertrags über die Europäische Union (EUV) nach wie vor fundamentaler Daseinszweck der Union.

Basierend auf dem institutionellen Erfahrungsschatz der Europäischen Kommission, der gelegentlichen Theaterdonner und pragmatische kleine Schritte produktiv verbindet, ist es gelungen, die EU als Vorreiter im globalen Kampf gegen den Klimawandel zu positionieren. Bei genauerer Betrachtung wird jedoch klar, dass das Erreichen der klimapolitischen

4 Ursula von der Leyen: Rede zur Eröffnung der Plenartagung des Europäischen Parlaments. Straßburg, 16. Juli 2019. 
Ziele bis 2050 ohne radikalen Kurswechsel wenig realistisch erscheint. Im energiepolitischen Zieldreieck aus Energieversorgungssicherheit, Klima- und Umweltschutz sowie Wirtschaftlichkeit dominieren die Interessen der Mitgliedstaaten. Dies ist einerseits der Tatsache geschuldet, dass Energie als strategisches Gut betrachtet wird und im Konfliktfall alle Parteien Autarkie gegenüber Interdependenz bevorzugen. Andererseits sind die unterschiedlichen Energieimport- und Produktionsstrukturen allzu verständlicher Auslöser von Interessenkonflikten. Die Strategie der Kommission, den Energiebinnenmarkt ins Zentrum zu rücken, flankiert von Regulierungsexport und technologischem Fortschritt, erweist sich in dieser Gemengelage als sinnvoll, da kleine pragmatische Schritte von den Mitgliedstaaten bevorzugt werden. Inwieweit dieser allerdings die europäische Energiesicherheit in einem umfassenden Verständnis befördert, ist fraglich.

\section{Europas Energiesicherheit: Definitionen und Herausforderungen}

Die am 25. Februar 2015 angenommene Rahmenstrategie zur Europäischen Energieunion setzt ehrgeizige Ziele und fasst jahrzehntealte Projekte zusammen: Eine Erhöhung der europäischen Energiesicherheit, ein vollständig integrierter europäischer Energiebinnenmarkt, erhöhte Energieeffizienz, Dekarbonisierung der Wirtschaft und verstärkte Forschungsanstrengungen werden genannt. ${ }^{5}$ Die nationalen Energie- und Klimapläne für den Zeitraum 2021 bis 2030 müssen diese fünf Ziele integrieren und zu ihrer Erreichung beitragen. Die Europäische Kommission veröffentlicht diesbezüglich regelmäßig Fortschrittsberichte, zuletzt am 9. April 2019. ${ }^{6}$ Zwar notiert dieser letzte Bericht insgesamt Fortschritte in einem Politikbereich, der in den vergangenen Jahrzehnten zu geringe Anstrengungen der Mitgliedstaaten erfahren hat. Allerdings zeigen ein Blick in die öffentlichen Statistiken zur Energieabhängigkeit und die globale geostrategische Lage die Notwendigkeit deutlich vermehrter Bemühungen. Und diese notwendigen Bemühungen zeigen wiederum nachdrücklich die Grenzen des Möglichen auf: Die stark divergierenden Interessen der Mitgliedstaaten bezüglich ihres jeweiligen nationalen Energiemixes, die schwache geostrategische Position der EU sowie die dringend erforderlichen klimapolitischen Maßnahmen zur Erreichung der EU-Klimaziele für 2030 und 2050 erschweren den Weg zu einem energiesicheren Europa. Um in allen Bereichen Erfolg zu haben, genügt es nicht, lediglich die Importabhängigkeit bei Erdgas, Öl und Uranbrennstäben $\mathrm{zu}$ verringern und erneuerbare Energiegewinnung verstärkt zu verfolgen. Vielmehr ist ein Umdenken energiepolitischer Prämissen notwendig: von einseitiger Abhängigkeit zu Interdependenz auch in der Planung zukünftiger Energiebeziehungen, von politischen Vorgaben zu unabhängiger Regulierung und von industriefokussierten Maßnahmen zu gesellschaftspolitischen Veränderungen sowie Lenkungsmaßnahmen.

5 Siehe Europäische Kommission: Mitteilung der Europäischen Kommission an das Europäische Parlament, den Rat, den Europäischen Wirtschafts- und Sozialausschuss, den Ausschuss der Regionen und die Europäische Investitionsbank. Rahmenstrategie für eine krisenfeste Energieunion mit einer zukunftsorientierten Klimaschutzstrategie, $\operatorname{COM}(2015) 80$ final.

6 Siehe Europäische Kommission: Bericht der Kommission an das Europäische Parlament, den Rat, den Europäischen Wirtschafts- und Sozialausschuss, den Ausschuss der Regionen und die Europäische Investitionsbank. Vierter Bericht zur Lage der Energieunion, COM(2019) 175 final. 
Studien zur Energiesicherheit rückten in den vergangenen Jahren in den Fokus akademischer Aufmerksamkeit. ${ }^{7}$ Think-Tanks, Energieversorger, der Weltenergierat, die 1975 als Reaktion auf die erste Ölkrise gegründete Internationale Energieagentur sowie zahllose politische Akteure haben die Literatur um Definitionsversuche bereichert. ${ }^{8}$ Klar ist, dass das Konzept der Energiesicherheit kontextabhängig, intersubjektiv, ${ }^{9}$ „polysemic“ (mehrdeutig) ${ }^{10}$ und „multi-dimensional“11 ist und über die Jahre hinweg eine konzeptionelle Ausdehnung um die Bereiche der Klimapolitik, Nachhaltigkeit und Energieeffizienz erfahren hat. ${ }^{12}$ Die Kontextabhängigkeit bezieht sich auf den Industrialisierungsgrad eines Landes, Risikoperzeptionen, Redundanzsysteme, Verbrauchsmuster, Infrastruktur etc. Einige Autoren plädieren für ein enges Verständnis von Energiesicherheit im Sinne von Energieversorgungssicherheit, ${ }^{13}$ während andere umfassende Definitionen vorlegen, die Einwirkungen auf die sozioökonomische Struktur einbeziehen. ${ }^{14}$ Welche Auswirkungen die Verfolgung des umfassenden Ziels der Energiesicherheit auf zwischenstaatliche Beziehungen hat, blieb bisher relativ unbeleuchtet.

Umfassende Energiesicherheit kann als das Zusammenspiel von zumindest acht Faktoren definiert werden: (1) Verfügbarkeit von Energie oder geografische und geopolitische Faktoren; (2) vorhandene und geplante Infrastruktur; (3) stabile/volatile Energiepreise; (4) gesellschaftliche Effekte; (5) Umwelt- und klimapolitische Aspekte; (6) Regelung, Regelungsdichte und -intensität (Governance); (7) Energieeffizienz und (8) zwischenstaatliche Beziehungen. Ziel der Energiesicherheitspolitik muss die „low vulnerability of vital energy systems"15 (d.h. die geringe Verwundbarkeit zentraler Energieversorgungssysteme) sein.

\section{Die Grundlage: geopolitische Faktoren}

Geopolitische Faktoren, nationaler Energiemix und Diversität der Energielieferanten und -quellen wirken sich direkt auf die Verfügbarkeit von Energie aus. Importunterbrechungen sollen durch Diversifikation möglichst verhindert werden. Angesichts der Dauer von Upstream-Verfahren sowie angesichts geopolitischer Verwerfungen oder Konflikten ist die möglichst breite und kostengünstige Streuung von Lieferant, Quelle und Transportweg eine Möglichkeit, die ununterbrochene Versorgung zu gewährleisten. Eine möglichst breite,

7 Siehe Beng Wah Ang/Wei Liang Choong/Tsan Sheng Adam NG: Energy Security: Definitions, dimensions and indexes, in: Renewable and Sustainable Energy Reviews, Februar 2015, S. 1077-1093.

8 Siehe Benjamin K. Sovacool: Introduction: Defining, measuring, and exploring energy security, Table I.1, in: Benjamin K. Sovacool (Hrsg.): The Routledge Handbook of Energy Security, Abingdon/New York 2011, S. 142, hier S. 3-6.

9 Jessica Jewell/Elina Brutschin: The Politics of Energy Security, in: Kathleen J. Hancock/Juliann Allison (Hrsg.): The Oxford Handbook of Energy Politics, Oxford, im Erscheinen.

10 Lynne Chester: Conceptualising Energy Security and Making Explicit its Polysemic Nature, in: Energy Policy 2/2010, S. 887-895.

11 Vlado Vivoda: Evaluating energy security in the Asia-Pacific region: A novel methodological approach, in: Energy Policy 9/2010, S. 5258-5263.

12 Felix Ciută: Conceptual Notes on Energy Security: Total or Banal Security?, in: Security Dialogue 2/2010, S. 123-144.

13 Aldo Spanjer: Russian Gas Price Reform and the EU-Russia Gas Relationship: Incentives, Consequences and European Security of Supply, in: Energy Policy 5/2007, S. 2889-2898; Tooraj Jamasb/Michael Gerald Pollitt: Security of supply and regulation of energy networks, in: Energy Policy 12/2008, S. 4584-4589.

14 Vivoda: Evaluating energy security, 2010.

15 Aleh Cherp/Jessica Jewell: The concept of energy security: Beyond the four As, in: Energy Policy, Dezember 2014, S. 415-421, hier S. 420. 
sich an natürlichen Ressourcen orientierende Diversität der Energieproduktion ist eine weitere Dimension der Verfügbarkeit. Im Jahr 2017 produzierten die EU-Mitgliedsländer ca. 45 Prozent der benötigten Energie selbst. ${ }^{16}$ Besonderes Augenmerk auf die Importstrukturen zu legen, erscheint daher sinnvoll. Europas Liefer- und Distributionsnetzwerk in den Bereichen Öl, Gas und Flüssiggas („liquefied natural gas“, LNG) ist nach Fertigstellung der Pipeline „Nord Stream 2“ weitestgehend ausgebaut. Mittelfristige Investitionen werden für den Ausbau der West-Ost-Gastransportkapazitäten notwendig, befinden sich doch im Westen Europas auch die meisten Flüssiggasanlandeterminals. Die Ostsee-Pipeline-Anbindungsleitung (OPAL) und die Europäische Gas-Anbindungsleitung (EUGAL) sind für den Weitertransport des in Greifswald anlandenden Gases von großer Bedeutung. Sorgenkinder bleiben Bulgarien und Griechenland, deren Anbindung an das europäische Netzwerk suboptimal ist. In naher Zukunft sollte daher vor allem Südosteuropa besonderes Augenmerk gewidmet werden. Die im Winter 2019 getroffene und für fünf Jahre gültige Vereinbarung zwischen der russischen Gazprom und der ukrainischen Naftogaz sieht jährliche Mindesttransportmengen vor. Statt der bisher rund 90 Milliarden Kubikmeter im Jahr sollen 2020 nur 65 Milliarden Kubikmeter russisches Gas durch die Ukraine nach Europa gepumpt werden. Von 2021 bis 2024 sind 40 Milliarden Kubikmeter Gas pro Jahr für den Transit geplant. Es ist anzunehmen, dass sich diese Transitmenge in Zukunft weiter verringern dürfte. An einem Ausbau und der Nutzung der westukrainischen Gasspeicheranlagen sollte Europa besonderes Interesse zeigen.

\section{Infrastruktur: Marktlogik vs. Politik}

Eine robuste Infrastruktur umfasst Distributionsnetzwerke, Raffinerien, Kraftwerke, Speicherstätten, Pipelines und geeignete, zunehmend digitale Kontroll- und Lenkungsmöglichkeiten. Angesichts der Vulnerabilität moderner Energienetzwerke rückt der Schutz der Anlagen vor Cyberkriminalität zunehmend in den Fokus. ${ }^{17}$ Die Resilienz der Infrastruktur ist ein Gradmesser für die perzipierte Sicherheit. In der EU sind der alle Kapazitätserfordernisse übersteigende Ausbau von Flüssiggasterminals und der unzureichende Ausbau von Interkonnektoren, z.B. zwischen Bulgarien und Rumänien, beredtes Zeugnis mangelnder Koordination. ${ }^{18}$ Weder die notwendigen Mengen an LNG am Markt noch die Transportkapazitäten mittels LNG-Schiffen sind vorhanden. Angesichts der geringen Förderkosten russischen Gases ist zudem die Wirtschaftlichkeit von LNG, von der Umweltverträglichkeit ganz zu schweigen, in Zweifel zu ziehen. Infrastrukturprojekte innerhalb der EU folgen derzeit weniger einer Marktlogik oder einem koordinierten strategischen Ziel, sondern sind Ergebnis politischer Interessen. Als Beispiele mögen die Gasleitung „Bulgaria-Romania-Hungary-Austria“ (BRUA) und die Baltic Pipe "Polen-Dänemark-Nordsee“ dienen. Der Plan von ExxonMobil, Gas aus den Förderstätten im Schwarzen Meer mittels BRUA auf den westeuropäischen Markt zu bringen, wurde durch den Rückzug des ungarischen Übertragungsnetzbetreibers unterminiert. Als Grund für diese Entscheidung darf

16 Siehe Europäische Kommission: EU energy statistical pocketbook and country datasheets. EU energy in figures, abrufbar unter: https://ec.europa.eu/energy/en/data-analysis/energy-statistical-pocketbook (letzter Zugriff: 9.1.2020).

17 Siehe Kim Zetter: Countdown to Zero Day. Stuxnet and the Launch of the World's First Digital Weapon, New York 2014.

18 Siehe Europäische Kommission: Vierter Bericht zur Lage der Energieunion, 2019. 
vermutet werden, dass die Nähe zwischen dem ungarischen Ministerpräsidenten Viktor Orbán und dem russischen Präsidenten Wladimir Putin genutzt wurde, um alternative Gasimportrouten zu verhindern. Eine Folge davon sind der Rückzug von ExxonMobil aus dem rumänischen Gasfördergeschäft und „stranded investments“ (verlorene Investitionen) in der Höhe von mehreren hundert Millionen Euro europäischer Fördergelder. Selbiges gilt für die Baltic Pipe: Anstelle der optimalen Nutzung vorhandener Kapazitäten wird eine neue Pipeline gebaut, die dazu dient, teures norwegisches Gas anstelle günstigen russischen Gases oder an Spotmärkten gehandeltes Gas zu importieren. Der Schutz der eigenen Gasindustrie war dabei wichtiger als der Schutz der Konsumenten. Vordergründig wird die Pipeline durch die Reduktion der Importabhängigkeit von russischem Gas legitimiert eine Abhängigkeit, die nicht besteht, würde doch bei Öffnung des polnischen Gasmarktes die Versorgung mit Gas aus den europäischen Spotmärkten zu kompetitiven Preisen möglich sein. Zu guter Letzt ist das Verhältnis zwischen Bulgarien und Rumänien eindeutiger Ausdruck politischen Versagens: Rumänien könnte problemlos Nettogasexporteur sein und einen südosteuropäischen Gashub begründen. Voraussetzung dafür ist logischerweise der Wille, Gas zu exportieren. Die viel zitierten technischen Probleme des rumänischen Unternehmens TRANSGAZ bei der zum Gastransport notwendigen Druckerhöhung in der Pipeline sind willkommene Ausrede für den Wunsch, Exporte zu limitieren. Die Tatsache, dass TRANSGAZ im staatlichen Eigentum steht und einer hohen Dividende der Vorzug vor notwendigen Investitionen gegeben wird, machte Rumänien zum Gasnettoimporteur. Anstelle der von der Europäischen Kommission angedachten neuen Verordnungen sollte daher wohl zuerst an die Umsetzung der vorhandenen Liberalisierungspakete gedacht werden. Unabhängige Regulierungsbehörden sind dazu unerlässlich.

\section{Stabile Energiepreise}

Energiepreise bestimmen die Leistbarkeit der Ressource. Wünschenswert sind eine geringe Volatilität und vor allem ein kompetitiver Markt, der den Handel mit Energieprodukten aller Art zum Vorteil des Energiekunden unter den Bedingungen des Umweltschutzes garantiert. In der EU ist der funktionierende Handel mit Gas im Nordwesten eine Garantie für die Versorgungssicherheit - weniger optimal stellt sich die Lage im Südosten Europas dar. Zwar wurden durch Investitionen in die Infrastruktur potenzielle Versorgungsengpässe vermieden, die Monopolstellung einiger Versorger wirkt sich jedoch suboptimal auf den Markt aus. Die Entwicklung der Gas- und Strompreise für Haushalte in der EU zeigt in den letzten zehn Jahren eine moderate, aber stetige Steigerung. Dabei gilt es wie immer, regionale Unterschiede und Unterschiede in der Besteuerung von Energie zu bedenken: Stiegen z.B. die Gaspreise in Irland zwischen 2017 und 2018 um 17,3 Prozent, gefolgt von Bulgarien (16,5 Prozent), Schweden (16,4 Prozent) und Rumänien (16,3 Prozent), so sanken sie in Kroatien (2,5 Prozent), Portugal (1,9 Prozent), Ungarn (0,4 Prozent) und Deutschland (0,2 Prozent). ${ }^{19}$ Unabhängige Regulatoren sind eine Grundvoraussetzung für eine transparente Preisgestaltung. Eine Stärkung der jeweiligen Regulationsnetzwerke (z.B. der Agentur für die Zusammenarbeit der Energieregulierungsbehörden oder des

19 Siehe Eurostat: Energiepreise im Jahr 2018. Energiepreise für Haushalte in der EU gegenüber 2017 gestiegen, Pressemitteilung, 21. Mai 2019, 88/2019. 
European Network of Transmission System Operators for Electricity) sollte von der Kommission in Angriff genommen werden.

\section{Bekämpfung der Energiearmut}

Energiearmut ${ }^{20}$ ist auch in den industriellen Gesellschaften des Westens ein immer ernster zu nehmendes Problem. Gesellschaftliche Effekte betreffen auch die Akzeptanz von Großprojekten im Leitungsbereich und Kraftwerksbau. Nach Angaben der Europäischen Kommission sind bis zu 54 Millionen EuropäerInnen von Energiearmut betroffen. ${ }^{21}$ Die Kombination von geringen Einkommen, schlechter Infrastruktur, minderer Qualität von Gebäuden und hohen Energiepreisen führt zu inakzeptablen Belastungen. ${ }^{22}$ Die Europäische Kommission schlug eine Reihe von Maßnahmen vor, darunter die Steigerung der Gesamtenergieeffizienz von Gebäuden, ${ }^{23}$ eine Energie-Governance ${ }^{24}$ sowie ein Monitoring durch die Mitgliedstaaten in den nationalen Energie- und Klimaplänen. ${ }^{25}$ Angesichts der steigenden Anzahl der Betroffenen forderte der Europäische Ausschuss der Regionen (AdR) in einer einstimmigen Stellungnahme ${ }^{26}$ zu verstärkten Maßnahmen auf. Diese Maßnahmen - und hier folgt der AdR dem Europäischen Parlament - sollen auch den Eingriff der Mitgliedstaaten in den Energiemarkt im Falle benachteiligender Strukturen zulassen. Der Bericht des Europäischen Energienetzwerkes „Energy Poverty in the European Union“ vom Januar 2019 veranschaulicht die Energiearmut vor allem in Süd- und Südosteuropa. ${ }^{27}$

\section{Umwelt- und Klimaschutz: Conditio sine qua non}

Umweltverschmutzung ist bei dem heutigen technologischen Stand ein unvermeidbares Nebenprodukt der Energieproduktion. Klar ist, dass der Ausstoß von $\mathrm{CO}_{2}$ und anderer klimaschädlicher Gase drastisch reduziert werden muss. Selbiges gilt für die Vermeidung von umweltschädlicher Förderung fossiler Energie, insbesondere Kohle. ${ }^{28}$ Dies kann durch technologische Neuerungen erreicht werden, deren Finanzierung in der politischen Diskussion aber zumeist ausgeblendet wird. Viel diskutiertes Beispiel ist die $\mathrm{CO}_{2}$-Abscheidung und -Speicherung. Angesichts der ambitionierten Ziele Europas (wie die Negativemissio-

20 Zur Definition siehe Europäische Kommission, EU Energy Poverty Observatory: What is energy poverty?, abrufbar unter: https://www.energypoverty.eu/about/what-energy-poverty (letzter Zugriff: 28.11.2019).

21 Siehe Steve Pye/Audrey Dobbins: Energy poverty and vulnerable consumers in the energy sector across the EU: analysis of policies and measures, INSIGHT_E: Policy Report 2, Mai 2015.

22 Siehe Lefkothea Papada/Dimitris Kaliampakos: A Stochastic Model for energy poverty analysis, in: Energy Policy, Mai 2018, S. 153-164.

23 Siehe Richtlinie (EU) 2018/844 des Europäischen Parlaments und des Rates vom 30. Mai 2018 zur Änderung der Richtlinie 2010/31/EU über die Gesamtenergieeffizienz von Gebäuden und der Richtlinie 2012/27/EU über Energieeffizienz, in: Amtsblatt der EU, Nr. L 156 vom 19. Juni 2018, S. 75-91.

24 Siehe Verordnung (EU) 2018/1999 des Europäischen Parlaments und des Rates vom 11. Dezember 2018 über das Governance-System für die Energieunion und für den Klimaschutz, zur Änderung der Verordnungen (EG) Nr. 663/2009 und (EG) Nr. 715/2009 des Europäischen Parlaments und des Rates, der Richtlinien 94/22/EG, 98/70/EG, 2009/31/EG, 2009/73,/EG, 2010/31/EU, 2012/27/EU und 2013/30/EU des Europäischen Parlaments und des Rates, der Richtlinien 2009/119/EG und (EU) 2015/652 des Rates und zur Aufhebung der Verordnung (EU) Nr. 525/2013 des Europäischen Parlaments und des Rates, in: Amtsblatt der EU, Nr. L 328 vom 21. Dezember 2018, S. 1-77.

25 Siehe Europäische Kommission: Saubere Energie für alle Europäer, Luxemburg 2019.

26 Europäischer Ausschuss der Regionen: Multi-Level-Governance und sektorübergreifende Zusammenarbeit zur Bekämpfung der Energiearmut, Stellungnahme, 26./27. Juni 2019, ENVE-VI/038.

27 European Energy Network: EnR Position Paper on Energy Poverty in the European Union, Januar 2019.

28 Siehe Dieter Helm: The Carbon Crunch: How We're Getting Climate Change Wrong - and How to Fix it, New Haven/London 2012. 
nen zur Erreichung der für 2050 anvisierten Emissionsziele) ist die $\mathrm{CO}_{2}$-Speicherung unvermeidlich. Umweltverbände kritisieren jedoch die technologische Ausgereiftheit und die möglichen Gefahren der $\mathrm{CO}_{2}$-Lagerung. Aber selbst bei rapidem technologischen Fortschritt sind eine Änderung des individuellen Konsumverhaltens und die gesellschaftliche Lenkung mittels ökologischer Steuerreformen notwendig.

\section{Europäische Koordination}

Realistische vorausschauende Planung, Langzeitstrategien, Finanzierungssicherheit und Regelsicherheit tragen entscheidend zur Aufrechterhaltung der Energieversorgung bei. Politische Besetzungen von unabhängigen Regulierungsbehörden sind einer geeigneten Governance-Struktur ebenso abträglich wie die politische Einmischung in kommerzielle Projekte, so selbige die gesetzlichen Auflagen erfüllen. Das Beharren der Mitgliedstaaten auf den ihnen in Art. 194 des Vertrags über die Arbeitsweise der Europäischen Union (AEUV) zugestandenen Energieträgermixen ist angesichts der unterschiedlichen Ressourcen- und geografischen Lage zwar verständlich, wird aber die gemeinsame Entscheidungsfindung bei immer strikter werdenden EU-Zielen bei der Emissionsminderung, dem Ausbau der erneuerbaren Energieträger und der Energieeffizienz nicht einfacher machen. Die neue Governance-Verordnung für die Energieunion trägt dem bereits Rechnung. In naher Zukunft ist jenseits verstärkter nationaler Berichtspflichten allerdings wenig zu erwarten zu unterschiedlich sind die nationalen Interessenlagen. ${ }^{29}$

\section{Forschung und Entwicklung}

Energieeffizienz und Energieintensität sind Ausweis technologischen Fortschritts. Das Beharren der EU-Mitgliedstaaten auf dem Primat des nationalen Energiemixes machte und macht vor allem die Technologiepolitik zu einem bevorzugten Politikfeld gemeinsamen europäischen Handelns. Im Rahmen des Strategischen Energietechnologieplans (SET-Plan) wurden seit 2008 in Kooperation mit nationalen Forschungsprogrammen und Unternehmen rund 71,65 Milliarden Euro in die Grundlagenforschung investiert. ${ }^{30}$ Die Einbringung neuer Technologien aufgrund von Standardvorgaben kann als erfolgreiche Lenkungsmaßnahme bezeichnet werden, wenn sie auch durchaus auf Kritik, vor allem von Unternehmen, stößt. Selbiger kann durch den Ausbau adäquater Technologieförderinstrumente jedoch begegnet werden. Gegenwärtig belaufen sich die Ausgaben der Union für Forschung und Entwicklung im Energiebereich auf 3,188 Milliarden US-Dollar damit liegt Europa klar hinter den USA (7,148 Milliarden US-Dollar). ${ }^{31}$ Hinzu kommen in Europa nationale Forschungsbudgets. Eine bessere internationale Koordination der Forschungsanstrengungen wäre empfehlenswert.

29 Siehe Severin Fischer/Oliver Geden: Die europäische Energie- und Klimapolitik, in: Peter Becker/Barbara Lippert (Hrsg.): Handbuch Europäische Union, Wiesbaden, im Erscheinen.

30 Siehe Severin Fischer: Die Energiewende und Europa. Europäisierungsprozesse in der deutschen Energie- und Klimapolitik, Wiesbaden 2017.

31 Siehe International Energy Agency: Energy Technology RD\&D Budgets 2019, Oktober 2019, abrufbar unter: https://www.iea.org/reports/energy-technology-rd-and-d-budgets-2019 (letzter Zugriff: 9.1.2020). 


\section{Energiesicherheit als Thema internationaler Beziehungen}

Die Zentralität des Themas der Energiesicherheit bestimmt in immer größerem Ausmaß auch die Beziehungen zwischen Akteuren der internationalen Politik. Energiethemen werden in teils offenen, teils subtilen politischen Äußerungen zur Erreichung strategischer Ziele eingesetzt. ${ }^{32}$ Seien es der US-amerikanische Widerstand gegen Nord Stream 2, die unterschiedlichen Interpretationen der Sinnhaftigkeit der Pipeline in Deutschland und in Polen, ${ }^{33}$ die Beziehungen zu Russland seit Ausbruch der Ukrainekrise, ${ }^{34}$ die Tätigkeit US-amerikanischer Konzerne im Schwarzen Meer, die Aufkaufversuche der staatlichen chinesischen Ölgesellschaft oder die russisch-türkische Energiekooperation: Feststellbar ist eine zunehmende Versicherheitlichung der Energiepolitik, im Zuge derer immer öfter die eigentlichen kompatiblen Interessen - Nachfragesicherheit und Versorgungssicherheit ${ }^{35}$ zur Erreichung strategischer Ziele umgangen werden. Die Schwierigkeiten bei der Durchleitung russischen Gases durch die Ukraine seit 2005 können als Beispiel dienen. Sie zeigen auch, dass der Typus des Energietransitstaates der Beziehung Produzent/Konsument oder Exporteur/Importeur hinzugefügt werden muss.

Aufgabe der Politik ist es, diese Faktoren zu einer Gesamtstrategie zu verbinden. Dabei auftretende Schwierigkeiten werden u.a. darauf zurückgeführt, dass daraus konkurrierende gleichwertige Ziele resultieren, zusammengefasst unter dem Stichwort „energy trilemma“"36. In Anlehnung an die Volkswirtschaft und angesichts der gestiegenen Komplexität kann man auch vom „magischen Vieleck“ der Energiepolitik sprechen. Energiesicherheit, Umweltverträglichkeit und Wettbewerb zeichnen sich durch eine gewisse Inkongruenz aus. Für die Wettbewerbsfähigkeit eines Landes ist die Bereitstellung günstiger Energie wichtig. Gleichzeitig fordert die Bevölkerung sichere und saubere Energie, deren Bereitstellung einen höheren Kostenaufwand bedeutet und neue Infrastrukturinvestitionen erfordert. Die Kohleförderung Polens liefert ein anschauliches Beispiel: Die Abneigung gegen eine zu hohe Abhängigkeit von russischem Gas und der Wunsch nach relativ billiger heimischer fossiler Energie konkurrieren mit dem Ziel der $\mathrm{CO}_{2}$-Reduktion und der Eindämmung anderer Umweltunverträglichkeiten des Kohlebergbaus. Ein anderes Beispiel ist Österreich: Mit einem relativ hohen Anteil an hydroelektrischen Kraftwerken am Energiemix des Landes kommt man dem Wunsch nach sauberer Energie nach - jeglicher weiterer Ausbau stößt jedoch an die Grenzen des ökologischen Landschaftsschutzes. Als ein allgemeines Beispiel lässt sich die Herausforderung der Intermittenz anführen: Selbstverständlich können erneuerbare Energien die Importabhängigkeit reduzieren. Zur Absicherung gegenüber wetterbedingten Produktionsproblemen sind jedoch herkömmliche Kraftwerke notwendig. Die hohen Investitionsanforderungen und Erhaltungskosten treffen zudem auf das Erfor-

32 Siehe die These vom ,interventionist paradigm“, vgl. Andreas Goldthau: Introduction: Policy Agendas for the Future of Global Energy, in: Global Policy 2/2012, S. 182-183.

33 Siehe Andreas Heinrich: Securitisation in the Gas Sector: Energy Security Debates Concerning the Example of the Nord Stream Pipeline, in: Kacper Szulecki (Hrsg.): Energy Security in Europe. Divergent Perceptions and Policy Challenges, Basingstoke 2018, S. 61-91.

34 Siehe dazu Kacper Szulecki/Kirsten Westphal: Taking Security Seriously in EU Energy Governance: Crimean Shock and the Energy Union, in: Kacper Szulecki (Hrsg.): Energy Security in Europe. Divergent Perceptions and Policy Challenges, Basingstoke 2018, S. 177-202.

35 Siehe statt vieler Daniel Yergin: Energy Security in the 1990s, in: Foreign Affairs 1/1988, S. 110-132.

36 World Energy Council: World Energy Trilemma 2012: Time to get real - the case for sustainable energy policy, London 2012. 
dernis der Redundanz, d.h. traditioneller Kraftwerke, die Schwankungen schnell ausgleichen können.

\section{Strategisch bedenkliche Energieabhängigkeit oder Interdependenz?}

Seit 2006 - dem Jahr der ersten Unterbrechungen von Gaslieferungen aus Russland durch die Ukraine in westeuropäische Hubs - ist eine, wie bereits erwähnt, zunehmende Versicherheitlichung der Debatte festzustellen. Das Schlagwort der „Energiewaffe“ Russlands, d.h. der Einsatz von Energielieferungen als außenpolitisches Instrument etc., dominiert seitdem die europäische Versorgungssicherheitsdebatte. Die Abhängigkeit Europas von russischen Gaslieferungen ist in den Importstatistiken nachzulesen: Die EU importiert insgesamt mehr als die Hälfte ihrer konsumierten Energie. 90 Prozent des verbrauchten Rohöls und 69 Prozent des Erdgases müssen importiert werden, was pekuniär mit ca. 1 Milliarde Euro pro Tag zu Buche schlägt. ${ }^{37}$ Die Abhängigkeit von einzelnen Lieferanten bzw. Transportwegen variiert je nach Mitgliedstaat und Energieträger. An einem Ende der Gesamtabhängigkeit rangieren Malta (100 Prozent), Zypern (96,2 Prozent) und Belgien (76 Prozent), am anderen Ende Länder wie Estland (6,8 Prozent) und Rumänien (22,3 Prozent). ${ }^{38}$ In der Folge soll Erdgas als Beispiel für die bestehende Abhängigkeit dienen, bestimmt es doch auch den öffentlichen Diskurs im Gefolge der Ukrainekrise und anderer geopolitischer Veränderungen (Stichwort Nordafrika). Im Jahr 2017 kamen 32 Prozent des importierten Gases aus Russland, gefolgt von 25 Prozent aus Norwegen, 9 Prozent kommen in Form von Flüssiggas aus den USA, Russland und Katar, 8 Prozent aus Nordafrika und 20 Prozent stammen aus heimischer Produktion. Letztere sinkt seit Jahren stetig: Der Rückgang zwischen 1990 und 2016 beträgt über 30 Prozent. Plakativ hierfür ist das Erdgasförderfeld Groningen in den Niederlanden. 2013 wurden dort noch 53,8 Milliarden Kubikmeter gefördert. Aufgrund seismischer Bedenken werden heute nur mehr ca. 20 Milliarden Kubikmeter gefördert. Seit dem Jahr 2017 sind die Niederlande daher Gasnettoimporteur. Ein Rückgang der Produktion ist auch in Großbritannien zu verzeichnen - bisher konnte dies nicht durch neue Förderungen in z.B. Dänemark oder Rumänien ausgeglichen werden. ${ }^{39}$

Die Streitigkeiten zwischen Russland und der Ukraine bezüglich Gaslieferungen führten im Jahr 2009 zu erheblichen Gasengpässen in Südosteuropa. Als Reaktion darauf verabschiedete die Europäische Kommission eine europäische Energiesicherheitsstrategie. ${ }^{40}$ Zehn Jahre nach den Lieferunterbrechungen ist es heute dank „reverse flows“, also der Möglichkeit, die Richtung des Gastransports in bestehenden Leitungen zu ändern, des Gasüberangebots, der etablierten Gashandelsplätze und vernünftiger Infrastrukturinvestitionen (auch in Gasspeicher) um Europas Energiesicherheit wesentlich besser bestellt. Die zentrale Frage bezüglich der Energieimporte lautet, wie die ca. 480 Milliarden Kubikmeter an Erdgas, die Europa heute jährlich verbraucht, garantiert werden können. Eine einfache Rechnung addiert die Pipelinekapazität in Europa und kommt zu dem Schluss, dass mehr

37 Siehe Europäische Kommission: Energy security strategy, 23. Oktober 2014, abrufbar unter: https://ec.europa.e u/energy/en/topics/energy-strategy-and-energy-union/energy-security-strategy (letzter Zugriff: 28.11.2019).

38 Siehe Europäische Kommission: EU Energy in figures. Statistical Pocketbook 2018, Luxemburg 2018, S. 66.

39 Siehe Europäische Kommission: EU energy statistical pocketbook.

40 Europäische Kommission: Mitteilung der Kommission an das Europäische Parlament und den Rat. Strategie für eine sichere europäische Energieversorgung, $\operatorname{COM}(2014) 330$ final. 
als genug Kapazitäten vorhanden sind - leider nur auf dem Papier. Das ukrainische Durchleitungsnetzwerk ist technisch veraltet und angesichts der unsicheren politischen Lage ist der Investitionswille börsennotierter Unternehmen mehr als begrenzt. Selbiges trifft auf die im Gesamtvolumen kleinen Exportmengen aus Nordafrika zu. Die Hoffnungen, dass LNG Pipelinegas („piped gas“) ersetzen kann, bewahrheiten sich nur in sehr geringem Maße. In der EU stehen derzeit 22 Regasifizierungsanlagen („LNG ports“) mit einem Volumen für 245 Milliarden Kubikmeter zur Verfügung. Ihre Auslastung liegt jedoch bei lediglich 10 Prozent. LNG kommt heute aus den USA, die enormen politischen Druck auf Europa ausüben, amerikanisches Flüssiggas russischem Pipelinegas vorzuziehen, aus Katar und aus Russland selbst. Jenseits der Frage der klimafeindlichen Produktion amerikanischen Flüssiggases („fracking“) sind die vorhandenen, limitierten Transportkapazitäten ebenso zu bedenken wie die geografischen und ökonomischen: Gas hat nicht nur die physikalische Tendenz, von hohem Druck zu niedrigem zu fließen, sondern auch von billigen zu teuren Märkten. Darüber hinaus ist die Infrastruktur, um das Gas vom funktionierenden Markt in Nordwesteuropa nach Südosteuropa zu transportieren, limitiert. Es bleibt der sogenannte südliche Korridor. Dieser umfasst den gescheiterten Versuch Russlands, einerseits mit der Pipeline "South Stream“ den ukrainischen Transit zu umgehen und andererseits Zugang zu den Ressourcen des Kaspischen Meeres zu erlangen. Das Ziel, mittels der Pipeline „Nabucco“ aserisches Gas durch Bulgarien, Rumänien und Ungarn bis zum wichtigen Central European Gas Hub in Baumgarten in Österreich zu führen, war ebenso ambitioniert wie aufgrund der politischen Uneinigkeit zwischen den beteiligten Staaten und nicht vollkommen transparenter Liefermengen aus Aserbaidschan unerreichbar. Turkmenisches, irakisches und langfristig auch iranisches Gas hätten zur Diversifizierung der Energieversorgung Europas fraglos beigetragen. Bevorzugt wurde schlussendlich die Transadriatische Pipeline (TAP), die Gas aus dem Transitland Türkei via Griechenland und Albanien nach Süditalien bringt.

Die vorhandenen Pipelineprojekte von der Transanatolischen Pipeline zur TAP, von Interkonnektoren durch Südosteuropa zu neuen, aber wohl kaum ökonomisch sinnvollen Pipelines wie „Tesla“ haben mangels Kapazität wenig Einfluss auf die Gesamtversorgungslage. Der Traum vom iranischen Gas trifft auf die harte Realität der Sanktionen, aber auch der technischen Gegebenheiten. Irans Gasindustrie und -infrastruktur benötigen zumindest eine Dekade zur Modernisierung. Die vor allem im Süden des Landes konzentrierten Gasfelder werden in erster Linie die stark steigende Binnennachfrage bedienen müssen, auch um das Regime zu stabilisieren. Die exportierbaren Gasreserven Aserbaidschans sind begrenzt; Gas aus Turkmenistan wird vorwiegend nach China exportiert und es fehlt die Transportinfrastruktur in den Westen. In beiden Fällen würde die sich als zunehmend schwieriger Partner verhaltende Türkei zum zentralen Verteilzentrum des Gases aus Zentralasien. Ungeklärt wäre weiterhin, wie das Erdgas von der türkischen Grenze in die EU gelangen soll. Die rumänische und bulgarische Infrastruktur kann und will dies offenbar aufgrund von politischer Einflussnahme nicht leisten.

Die Projekte in Reaktion auf die russisch-ukrainische Krise über den Gastransport konzentrieren sich daher auf eine neue Pipeline, die Russland und Deutschland direkt durch die Ostsee verbinden soll: Nord Stream 2. Über 1230 Kilometer lang sollen zwei parallele Stränge entlang der Route von Nord Stream 1 für ein zusätzliches Volumen 
von 55 Milliarden Kubikmetern sorgen. Technisch ist der Ausbau vergleichsweise simpel und kostengünstig. Das Gas würde in den nordeuropäischen Spotmärkten landen und von dort in ganz Europa verteilt werden. Die Widerstände gegen das Projekt sind umfassend, werden aber vor allem aus pekuniärem Interesse vorgebracht, da Transitstaaten an Einkommen verlieren. Auch geht es um die Protektion nationaler Energieversorger: Weder Polen noch Ungarn haben ihre Energiemärkte bisher in adäquater Weise geöffnet. Eine solche Öffnung würde es sehr leicht ermöglichen, Gas zu einem kompetitiven Preis auf den westeuropäischen Spotmärkten zu erwerben. Im Dezember 2019 verabschiedeten die USA Sanktionen gegen am Bau von Nord Stream 2 beteiligte Firmen. Dieser doch ungewöhnliche Akt zwischen den transatlantischen Partnern wird Nord Stream 2 nicht verhindern können, aber wohl um mindestens sechs Monate verzögern. Über die US-amerikanischen Motive kann nur spekuliert werden, da sie einer rationalen Analyse jenseits des Wunsches nach Abnahme von mehr amerikanischem Flüssiggas durch Europa kaum zugänglich sind. Die Klage über einen weiterhin zunehmenden Einfluss Russlands bzw. eine zunehmende Abhängigkeit von russischem Gas ist daher cum grano salis zu nehmen, hat sich Russland in den vergangenen mehr als 50 Jahren als verlässlicher Lieferant (mit konkurrenzlos niedrigen Preisen und frequenten Preisanpassungen in den langfristigen Lieferverträgen) erwiesen. Darüber hinaus haben mehrere Experten ${ }^{41}$ überzeugend dargelegt, dass Russland von einem Abbruch der energiewirtschaftlichen Beziehungen stärker getroffen wäre als Europa. 65 Prozent aller Erlöse des russischen Gasriesen Gazprom ergeben sich aus Exporten in die EU. ${ }^{42}$ Die wirtschaftliche Vernunft sollte die gegenseitige Abhängigkeit erkennen und produktiv nutzen. Die völkerrechtswidrige Annexion der Krim durch Russland und die anhaltenden Konflikte in der Ostukraine ziehen das Bild vom verlässlichen Lieferanten in Zweifel. Es stellt sich die Frage, inwieweit im Schatten der Interdependenz die Interessen mittelbar betroffener Staaten in Mitleidenschaft gezogen werden.

Dabei steht außer Zweifel, dass die Trias europäischer Energie-(Außen-)politik - Erhöhung der Energieeffizienz und des Anteils der erneuerbaren Energien, Diversifikation von Produzenten sowie Leitungen und Export von europäischen Standards und Regularien in angrenzende Regionen z.B. über die Organisation „Energy Community“43 - einer veränderten geopolitischen Lage gegenübersteht. Angesichts des 50-prozentigen Anteils der Energieexportwirtschaft am russischen Staatseinkommen sind die Energiebeziehungen aber auch ein wesentlicher stabilisierender Faktor. Im Lichte der globalen Klimakrise ist die Frage daher, wie dieser stabilisierende Faktor in einem post-fossilen Zeitalter transformiert werden kann. Die EU sollte sich in ihren Langzeitszenarien zum Umwelt- und Klimaschutz daher auch vermehrt Gedanken über die zukünftige Rolle Russlands machen. Ohne Zweifel wird auch Nord Stream 2 die Ukraine als Transitland nicht gänzlich überflüssig machen: Anstelle von 140 Milliarden Kubikmeter werden es in Zukunft aber wohl nur 30 bis 40 Milliarden Kubikmeter transportierten Gases sein. Die dem ukrainischen Staat dabei

41 Andreas Goldthau: Rhetoric versus reality: Russian threats to European energy supply, in: Energy Policy 2/2008, S. 686-692; Karen Smith Stegen: Deconstructing the "Energy Weapon": Russia's Threat to Europe as case study, in: Energy Policy 10/2011, S. 6505-6513.

42 Dem stehen 26 Prozent heimischer Verkäufe und 9 Prozent weiterer Exporte gegenüber. Siehe Gazprom Export: Delivery statistics. Gas supplies to Europe, abrufbar unter: http://www.gazpromexport.ru/en/statistics/ (letzter Zugriff: 9.1.2020).

43 Siehe Details zu der Organisation unter: https://www.energy-community.org/ (letzter Zugriff: 9.1.2020). 
entgehenden Durchleitungsgebühren müssen im Interesse einer Stabilisierung des Landes kompensiert werden. Dabei sollte Europa kurz- und mittelfristig die in der Westukraine vorhandenen Gasspeicheranlagen im europäischen Gesamtinteresse nutzen.

\section{Energiesicherheit erfordert Klimaschutz}

Ohne Zweifel sind die von der EU geförderten Vorrichtungen zur Umkehrung der Gasflüsse ein wesentlicher Beitrag zur Versorgungssicherheit der östlichen Mitgliedstaaten, aber auch der Ukraine. Auch die gestärkte Rolle der EU-Agentur für die Zusammenarbeit der Energieregulierungsbehörden (Agency for the Cooperation of Energy Regulators, ACER), die Koordinationsversuche bei grenzüberschreitenden Infrastrukturvorhaben und die erhöhte Transparenz bei der Nutzung von Erdgasspeichern sind wichtige Schritte auf dem Weg zu einer größeren Energieversorgungssicherheit. Energiesicherheit bedeutet aber weit mehr als lediglich die Sicherung oder Verringerung von Energieimporten. Wichtiges Element einer Energiesicherheitsstrategie muss der Klimaschutz sein. Es geht um nichts Geringeres als eine zukunftsgerichtete Energie- und Klimaschutzpolitik, die klare Endziele definiert, Übergangsphasen dekliniert und massiv in Forschung investiert. Der bis 2030 vereinbarte Ausbau erneuerbarer Energie, basierend auf freiwilligen Selbstverpflichtungen, wird nicht ausreichen, die bereits heute dramatische Veränderung des Klimas zu mitigieren. Die zeitgemäße Frage kann daher nur heißen: Wie kann Energiesicherheit unter den Bedingungen des Klimawandels gewährleistet werden? Neue Gasförderstätten, neue Pipelines, mehr Windkraft und erhöhte Energieeffizienz werden nicht ausreichen, umfassende Energiesicherheit zu gewährleisten.

Europa wird sich der Frage stellen müssen, wie die für 2050 anvisierte $\mathrm{CO}_{2}$-Neutralität $\mathrm{zu}$ erreichen ist. Die Bepreisung der $\mathrm{CO}_{2}$-Emissionen kann sowohl über eine $\mathrm{CO}_{2}$-Steuer als auch über die Ausweitung des Emissionshandels erfolgen. Frans Timmermans, in der neuen Kommission für den Klimaschutz zuständig, hatte sich für eine $\mathrm{CO}_{2}$-Steuer eingesetzt. Die Europäische Volkspartei befürwortet dagegen die Ausweitung des Emissionshandels (z.B. die Ausdehnung auf den bis dato exkludierten Transportsektor). Ein Grund für diese Bevorzugung dürfte in der Umsetzbarkeit liegen, wird dafür doch nur eine einfache Mehrheit im Rat der EU benötigt. Die Einführung einer $\mathrm{CO}_{2}$-Steuer bedarf dagegen der Zustimmung aller Mitgliedsländer.

Die EU hat sich das Ziel gesetzt, bis 2050 die Treibhausgasemissionen um 80 bis 95 Prozent zu reduzieren. Das Zwischenziel für 2030 sind eine Reduktion um 40 Prozent bei EU-internen Emissionen im Vergleich zum Jahr 1990 sowie eine Steigerung der Nutzung erneuerbarer Energien auf 30 Prozent des gesamten Energieverbrauches. Die anvisierte Treibhausgas-Neutralität stößt bei den Mitgliedstaaten nicht auf ungeteilte Zustimmung, wäre aber eine logische Fortsetzung des bisher Erreichten: Seit 1990 hat die EU ihre Treibhausgasemissionen um 23 Prozent gesenkt. Die legislative Arbeit mündete 2018 in 
mehreren Richtlinien (z.B. ETS-Richtlinie ${ }^{44}$, LULUCF-Verordnung ${ }^{45}$ ), die zur Erreichung des EU-Klimaziels 2030 beitragen. Allerdings müssen nach einem Bericht des Intergovernmental Panel on Climate Change ${ }^{46}$ die weltweiten Emissionen auf null sinken, um den Zielkorridor einer Erderwärmung von 1,5 bis $2^{\circ} \mathrm{C}$ zu erreichen. Unvermeidbare Emissionen müssten dabei mit negativen Emissionen ausgeglichen werden. Die Ende 2018 von der Europäischen Kommission vorgelegte langfristige Klimastrategie ${ }^{47}$ schlägt vor, das Treibhausgasreduktionsziel 2050 von derzeit 80 bis 95 Prozent auf 100 Prozent zu erhöhen. Da es sich lediglich um einen Vorschlag der Kommission handelte, konnte der Rat der EU seine eigenen Vorstellungen entwickeln. Jedoch gelang es beim Treffen des Europäischen Rates im Juni 2019 nicht, einen Konsens herzustellen - zu groß war der Widerstand aus Polen, Tschechien, Ungarn und Estland. Zahlreiche Mitgliedstaaten haben bereits nationale Nullemissionsziele bis 2050 verabschiedet (z.B. Schweden und Frankreich) und 22 Staaten haben sich hinter den Vorschlag der Kommission gestellt. Wie Oliver Geden und Felix Schenuit ${ }^{48}$ in ihrer Analyse feststellen, werden die entscheidenden Punkte die der Differenzierung zwischen den Mitgliedstaaten sowie die Bereitschaft, langfristige Klimaziele auch durch kurzfristige, man ist versucht zu schreiben wahlbeeinflussende, Maßnahmen zu implementieren, sein.

Die neue Präsidentin der Europäischen Kommission hat klargemacht, dass eine neue europäische Wirtschafts- und Industriepolitik die Ziele für $2050\left(\mathrm{CO}_{2}\right.$-Neutralität) legislativ berücksichtigen muss. ${ }^{49}$ Wird dieses Ziel ernst genommen, so müssen auch die Folgen eines „European Green Deal“ auf dem Arbeitsmarkt berücksichtigt werden. Die Etablierung eines Fonds zur Abfederung der Folgen ist daher ein wichtiger und richtiger Schritt, um regionale Disparitäten auszugleichen. Des Weiteren ist die Ankündigung der Europäischen Investitionsbank (EIB), zukünftig 10 Milliarden Euro für den Übergang zu einer nachhaltigen Kreislaufwirtschaft ${ }^{50}$ zur Verfügung stellen zu wollen, ein starkes Zeichen der Unterstützung für von der Leyens „Green Deal“. Damit einher geht eine Reduktion der EIB-Investitionsunterstützungen für fossile Energieträger in den Bereichen Exploration, Extraktion und Transport. Erdgas als notwendige Brückentechnologie könnte damit

44 Richtlinie (EU) 2018/410 des Europäischen Parlaments und des Rates vom 14. März 2018 zur Änderung der Richtlinie 2003/87/EG zwecks Unterstützung kosteneffizienter Emissionsreduktionen und zur Förderung von Investitionen mit geringem $\mathrm{CO}_{2}$-Ausstoß und des Beschlusses (EU) 2015/1814, in: Amtsblatt der EU, Nr. L 76 vom 19. März 2018, S. 3-27.

45 Verordnung (EU) 2018/841 des Europäischen Parlaments und des Rates vom 30. Mai 2018 über die Einbeziehung der Emissionen und des Abbaus von Treibhausgasen aus Landnutzung, Landnutzungsänderungen und Forstwirtschaft in den Rahmen für die Klima- und Energiepolitik bis 2030 und zur Änderung der Verordnung (EU) Nr. 525/2013 und des Beschlusses Nr. 529/2013/EU, in: Amtsblatt der EU, Nr. L 156 vom 19. Juni 2018, S. 1-25.

46 Siehe The Intergovernmental Panel on Climate Change: Global Warming of $1.5^{\circ} \mathrm{C}$, IPCC Sonderbericht, abrufbar unter: https://www.ipcc.ch/sr15/ (letzter Zugriff: 28.11.2019).

47 Europäische Kommission: Mitteilung der Kommission an das Europäische Parlament, den Europäischen Rat, den Rat, den Europäischen Wirtschafts- und Sozialausschuss, den Ausschuss der Regionen und die Europäische Investitionsbank. Ein sauberer Planet für alle. Eine Europäische strategische, langfristige Vision für eine wohlhabende, moderne, wettbewerbsfähige und klimaneutrale Wirtschaft, $\operatorname{COM}(2018) 773$ final.

48 Oliver Geden/Felix Schenuit: Klimaneutralität als Langfrist-Strategie. Die Ausgestaltung des EU-Nullemissionsziels und die Folgen für Deutschland, Stiftung Wissenschaft und Politik: SWP-Aktuell 38/2019.

49 Siehe Emmanuel Tuchscherer: Neutralité carbone en Europe: comment y parvenir?, Fondation Robert Schuman: Question d'Europe 519/2019.

50 Siehe Europäische Investitionsbank: Zehn Milliarden Euro für die Kreislaufwirtschaft in der EU, 18. Juli 2019, abrufbar unter: https://www.eib.org/de/press/all/2019-191-eur-10-billion-to-support-the-circular-economy-in -the-eu (letzter Zugriff: 28.11.2019). 
eine kürzere Lebensspanne haben, als von der Gasindustrie vorgesehen. Von der Leyens Vorschlag, 25 Prozent der EIB in eine Europäische Klimaschutz-Bank umzuwandeln, und die Ankündigung Christine Lagardes, der Europäischen Zentralbank mehr „Green Bonds“ zu verordnen (und damit von der bisherigen Neutralität Abstand zu nehmen), lassen auf ein umfassendes Konzept hoffen. Nicht alle Mitgliedsländer können oder wollen sich allerdings diesem Ziel verpflichtet sehen. Insbesondere die osteuropäischen Staaten pochen auf mehr nationale Eigenständigkeit. Dass Timmermans als Vizepräsident der Kommission und verantwortlich für Klimaschutz nicht die uneingeschränkte Unterstützung mancher zentral- und osteuropäischer Mitgliedstaaten genießt, mag eine weitere Herausforderung darstellen.

\section{Die EU auf dem Weg in ein post-fossiles Zeitalter}

Wiewohl sich die EU im Jahr 2020 einer gesicherten Versorgungslage gegenübersieht, sind die Herausforderungen der nächsten Jahrzehnte nicht zu übersehen. An erster Stelle steht dabei die Vereinbarkeit von steigendem Energiekonsum und Klima- und Umweltschutz. Eine europaweite $\mathrm{CO}_{2}$-Besteuerung ist hier ebenso wichtig wie eine koordinierte Forschungsanstrengung z.B. im Bereich der $\mathrm{CO}_{2}$-Sequestrierung. Der technologische Fortschritt allein wird allerdings ein Umdenken im Bereich Individualverkehr nicht überflüssig machen können. Im Bereich der Infrastruktur muss Europa eine geeignete Mischung zwischen Markt- und Versorgungsgarantie finden und eine konsequente Liberalisierung bzw. Umsetzung der Liberalisierungspakete verfolgen. Nicht die viel zitierte Souveränität des nationalen Energiemixes ist dabei das Problem, sondern die mangelnde europäische Kooperation und Koordination. Die Unterschiedlichkeit sollte als Vorteil gesehen werden, erhöht sie doch die Resilienz des Gesamtsystems. Ein weiteres prominentes Ziel der neuen Europäischen Kommission muss die wirksame Bekämpfung der Energiearmut sein. Die Einführung eines Überprüfungsmechanismus für länderspezifische Ziele und Aktionspläne plus Empfehlung durch den Rat der EU wäre zu überlegen. Bleibt Europas Verhältnis zu Russland: Während die Industrie auf die Marktlogik pocht, sehen politisch Verantwortliche mit großer Sorge auf die internationale Politik Russlands. Vom Ukrainekonflikt bis zur russischen Syrienpolitik, von der Einmischung in demokratische Wahlvorgänge bis zu neuen Rüstungsanstrengungen reichen die Themen. Bisher hat sich gezeigt, dass eine konsequente Haltung Europas und ein gesicherter Import von Energieressourcen einander nicht ausschließen. Angesichts der Devisenbedürfnisse Russlands und seiner schwach diversifizierten Wirtschaft ist von einer Änderung nicht auszugehen. Die EU muss aber bereits heute beginnen, über die Neuausrichtung ihres Verhältnisses zu Russland in einem post-fossilen Zeitalter nachzudenken. 